\title{
¿Cómo mujeres inmigrantes enfrentan la violencia en la relación de pareja?
}

\author{
Roberta de Alencar-Rodrigues \\ Escola de Psicologia da Faculdade Meridional \\ Leonor María Cantera Espinosa \\ Universidad Autónoma de Barcelona
}

\begin{abstract}
Resumen
Este estudio cualitativo tiene el objetivo de identificar las estrategias de las mujeres inmigrantes latinoamericanas para enfrentar la violencia de género en la pareja. Los datos obtenidos a través de entrevistas semi estructuradas indican que los recursos personales que facilitan corresponden a estrategias de enfrentamiento enfocadas en la resolución de problemas y emoción, mientras los factores que obstaculizan son el miedo, la vergüenza, la soledad y las creencias tradicionales. Se subraya que la decisión de salir de una relación de violencia de género en la pareja no depende solo de factores individuales y externos, pues aunque la mujer desee interrumpir la relación, resulta importante que ella perciba que tiene medios (apoyo social, apoyo institucional, recursos materiales) para concretar este objetivo.
\end{abstract}

Palabras clave: estilos de enfrentamiento; género; inmigrantes; mujeres; violencia.

\section{Resumo}

Como mulheres imigrantes enfrentam a violência de gênero? Este estudo qualitativo tem o objetivo de identificar as estratégias das mulheres imigrantes latino-americanas para enfrentar a violência de gênero na relação conjugal. Os dados obtidos através de entrevistas semiestruturadas indicam que os recursos pessoais que facilitam a redução ou o término da violência de gênero correspondem a estratégias de enfrentamento enfocadas na resolução de problemas e emoção, enquanto os recursos que obstaculizam são o medo, a vergonha, a solidão e as crenças tradicionais. Sublinha-se que a decisão de sair de uma relação de violência de gênero não depende somente de fatores individuais, pois apesar da mulher desejar interromper o relacionamento, é importante que ela perceba que tem meios (apoio social, apoio institucional, recursos materiais) para concretizar este objetivo.

Palavras-chave: estilos de enfrentamento; gênero; imigrantes; mulheres; violência.

\begin{abstract}
How immigrant women cope with gender violence? It is a qualitative study with the objective of identifying the strategies of Latin American immigrant women to cope with gender violence in couples. The qualitative data is gathered through semi-structured interviews and shows that personal resources that contribute the cessation or reduction of partner violence are related to coping strategies focused on solving problems and emotion, while resources that impede are fear, shame, loneliness and traditional beliefs. It highlights that the decision to leave an abusive relationship does not depend only on individual factors because although the women want to end the relationship, it's important that they realize that they can count with support (social support, institutional support, material resources) to achieve this objective.
\end{abstract}

Keywords: coping styles; gender; immigrants; violence; women.

a violencia de género en la pareja tiene lugar en todas las culturas, clases sociales, etnias, religiones, edades. Trata de la violencia que la mujer padece por el hecho de ser mujer y que produce sufrimiento físico, sexual o psicológico (Conferencias de las Naciones Unidas sobre la $\mathrm{Mu}$ jer, 1995). Esos efectos producidos por la violencia vulneran 
los derechos humanos; afectando así su pleno desarrollo. La violencia de género puede darse en las relaciones de pareja, en la edad infantil, en el ámbito laboral, a través de la mutilación genital femenina, el tráfico de mujeres y el matrimonio forzado. Para efectos de este trabajo, se enmarca en la violencia que las mujeres sufren en una relación heterosexual a manos de un hombre. Por ello, este estudio se hace eco de la definición de violencia de género en la pareja que se refiere al maltrato que se da en parejas heterosexuales de hombre a mujer, tal como se utiliza en la Ley $1 / 2004$.

Cortés y Serra (2011) recomiendan el término violencia de género en lugar de violencia familiar o doméstica, porque los últimos conceptos omiten el sexo de los actores y de las víctimas. Antes de caracterizar la violencia de género en la pareja, conviene aclarar la diferencia entre sexo y género. Sexo se refiere al hecho biológico, y según el Señas (2006), "es un conjunto de características del organismo que distinguen al macho de la hembra". El género, según Louro (1997), incide en la construcción social e histórica producida sobre las características biológicas. Desde este referente se entiende, según Heilborn (1998, p. 53), que "las concepciones sobre la diferencia sexual no eliminan el hecho de que existe una diferenciación en los cuerpos". Sin embargo, hay que hacer notar que las diferencias sexuales no excusan la desigualdad de género (Cantera, 2005). Por lo tanto, no son los atributos sexuales, sino la manera en la que ellos son interpretados, representados y valorados, lo que va a delimitar el concepto femenino o masculino en un determinado momento histórico.

Partiendo de la concepción del género como construcción cultural, se percibe la violencia en la pareja no como un problema de la naturaleza sexual de las relaciones entre macho y hembra, sino como un fenómeno histórico, producido y reproducido por las estructuras sociales de dominación de género y reforzado por la ideología patriarcal (Cantera, 2007). Dichas proposiciones demuestran que las tendencias de dominación no están inscritas en la naturaleza masculina, sino que se aprenden mediante la socialización (Alberdi, 2005), pues la cultura preexiste a las personas y desde el nacimiento impregna toda relación (Turintetto \& Vicente, 2008).

Cortés y Serra (2011) enumeran las siguientes características de la violencia de género en la pareja: intención, persistencia, vulnerabilidad, desigualdad de poder, imprevisibilidad, producción de estado de confusión. La violencia tiene lugar en un contexto de desequilibrio de poder (Cortés \& Serra, 2011) debido a las condiciones de vulnerabilidad en las que las víctimas se encuentran. Entre las situaciones de vulnerabilidad, las autoras ejemplifican el aislamiento social, la dependencia económica, el duelo por la pérdida de una persona significativa, la carencia afectiva, la dedicación plena a un hijo recién nacido y ser mujer en una sociedad machista. El estado de confusión tiene que ver con la sensación de inquietud y angustia debido al hecho de recibir malos tratos de una persona que le dice amarle (Cortés \& Serra, 2011).

Conviene aclarar los términos agresión, violencia y conflicto, pues muchas veces son abordados en la literatura como si fueran sinónimos. Según Cortés y Serra (2011) la agresión es un acto que emerge como actitud defensiva proporcional al ataque recibido. Aunque la agresión sea mutua, la víctima tiene menos probabilidades para defenderse.

La naturaleza del conflicto, según Cortés y Serra (2011), no es violenta. Se trata de una discusión o enfrentamiento mediante el diálogo, donde no deja de existir respecto hacia la libertad e integridad del otro. Conforme las autoras, en el conflicto en la pareja la relación es simétrica y no hay uso de las vulnerabilidades que se reconocen en el otro para hacer uso de la dominación.

Para efectos de este artículo se adopta la siguiente definición de violencia:

"La violencia es una agresión consciente, continuada y, habitualmente, selectiva contra la víctima, que toma las formas necesarias sutiles o evidentes, para conseguir su objetivo: el mantenimiento de la satisfacción de las necesidades de quien la ejerce" (Cortés \& Serra, 2011, p. 20).

La violencia genera una situación de estrés en la familia (Gadoni-Costa \& Dell’Aglio, 2011) con lo cual las mujeres utilizan diferentes estrategias para amenizar los daños hacia su integridad y seguridad física (Gadoni-Costa \& Dell'Aglio, 2009). Por lo que respecta al tema de este artículo, el estrés al que se refiere está relacionado a las exigencias externas (económicas y sociales) e internas, que son evaluadas como excedentes a los recursos de la persona.

Estas estrategias de enfrentamiento son llamadas de estilos de coping por Lazarus (1993). Primeramente este autor adoptó el término estrategia de enfrentamiento de estilo para referirse a las defensas de ego relacionadas con la preservación de la integridad psicológica según una perspectiva psicoanalítica. En los años 70, Lazarus (1993) pasó a llamarlas estrategia de enfrentamiento de proceso, ya que son esfuerzos cognitivos y conductuales para lidiar con el estrés que alteran a lo largo del tiempo y dependen del contexto donde tienen lugar. Así no hay estrategias buenas o malas dado que los esfuerzos invertidos por las mujeres dependen del contexto en que tiene lugar la violencia y que esos se modifican a medida que las amenazas cambian. Así Gadoni-Costa y Dell’Aglio (2009) afirman que las estrategias dependen de variables individuales (creencias y habilidades) y ambientales (apoyo social). Lazarus (1993) clasifica las estrategias de enfrentamiento de proceso en: (a) enfocada en el problema y (b) enfocada en la emoción.

La primera implica cambiar la relación problemática entre la persona y el ambiente a través de modificaciones en sí mismo o en el ambiente con el objetivo de manejar problemas específicos. Según Antoniazzi, Dell’Aglio, y Bandeira (1998) la acción puede ser dirigida internamente o externamente. Conforme esas autoras, la estrategia enfocada en el problema dirigida a la fuente externa de estrés incluye solucionar un conflicto interpersonal o pedir ayuda de otras personas, mientras la estrategia basada en el problema dirigido a la fuente interna consiste en redefinir el factor estresor.

La segunda función, enfocada en la emoción, consiste en alterar la relación estresante con el entorno (vigilancia o evitación) y el significado sobre lo que está pasando. Según 
Folkman, Lazarus, Gruen, y DeLongis (1986), las estrategias de enfrentamiento enfocadas en la emoción promueven la adaptación a situaciones estresantes ajenas al control de la persona como el caso de la violencia en relaciones de pareja, mientras las estrategias de enfrentamiento enfocadas en el problema facilitan el mejor ajuste cuando el individuo hace frente a un evento estresante que está dentro de su control. Según Gadoni-Costa y Dell'Aglio (2009), las mujeres pueden utilizar ambas estrategias dado que el enfrentamiento basado en el problema interacciona simultáneamente con el enfrentamiento basado en la emoción. Además Gadoni-Costa y Dell’Aglio (2011) señalan que la duración de la relación de pareja puede alterar las respuestas de enfrentamiento a lo largo del tiempo dado que si la mujer permanece mucho tiempo en la relación, ella pone todo su esfuerzo para que la relación tenga éxito.

Alencar-Rodrigues y Cantera (2013) señalan que las mujeres adoptan estrategias de enfrentamiento basadas en la resolución de problema como la búsqueda de ayuda en la red social y en las instituciones.

Hage (2006) analizó la vivencia de 10 mujeres que sufrieron violencia y expresaron haber buscado ayuda en la red social. Las participantes del estudio de Hage (2006) afirmaron que la falta de recursos institucionales a los que acudir para solicitar ayuda para la situación de violencia les hizo sentir atrapadas en la relación de dominio del agresor.

Alencar-Rodrigues y Cantera (2013) aclaran que la red social puede apoyar a la mujer que sufre violencia siempre que la red garantice el bienestar de las mujeres por medio del ofrecimiento de recursos materiales y de apoyo emocional, sin juicios.

Otra estrategia de enfrentamiento enfocada en el problema es el uso de la violencia como actitud de defensa (GadoniCosta \& Dell’Aglio, 2009). Resulta posible que las agresiones continúen si las mujeres asumen la forma de enfrentamiento a través del confrontamiento directo, pues el agresor puede aumentar sus esfuerzos para mantener el control a través de la violencia (Gadoni-Costa \& Dell'Aglio, 2011). Dentro de las estrategias enfocadas en la emoción, la reevaluación tiene lugar cuando las mujeres reevalúan aspectos importantes de sí mismas, de la pareja autora de la violencia y de la relación de pareja, poniendo límites en situaciones que les ponían tristes, nombrando el abuso y estableciendo autonomía en relación con su pareja (Zink, Jacobson, Pabst, Regan, \& Fisher, 2006). La vigilancia se refiere al estado de alerta a los estímulos del ambiente y la evitación, según Gadoni-Costa y Dell'Aglio (2009) tiene que ver con la represión de los recuerdos para evitar reflexionar sobre el evento violento. Estas estrategias pueden ser adaptativas cuando previenen que las situaciones de estrés aumenten (Waldrop \& Resick, 2004).

Hay que tener cuidado con las estrategias que no ponen fin a la situación de violencia, pues ello afecta la capacidad de las mujeres confiar en su capacidad de solucionar un problema y elegir estrategias de enfrentamiento (Gadoni-Costa \& Dell'Aglio, 2011). Costa y Dell'Aglio (2009) ponen el acento en la importancia de reforzar las estrategias de enfrentamiento en las intervenciones con mujeres para cuestionar creencias disfuncionales y desarrollar un nuevo repertorio de estrategias, pues ello les ayuda a superar los efectos de la victimización.

\section{Método}

Este trabajo aborda la violencia de género en la pareja contra mujeres inmigrantes latinoamericanas, pues la literatura apunta que las condiciones del contexto migratorio pueden aumentar la vulnerabilidad del maltrato. Con el ánimo de conocer más la relación entre el proceso migratorio y la violencia, se plantea un estudio cualitativo con el objetivo de identificar las estrategias de las mujeres inmigrantes latinoamericanas para enfrentar la situación de maltrato.

Participaron 14 mujeres inmigrantes latinoamericanas, mayores de 18 años, que han padecido violencia en la relación de pareja heterosexual y cumplen tres criterios: ser mujer inmigrante latinoamericana, haber sufrido violencia de género en la pareja en España y haber recibido ayuda por parte de algún servicio de la red de recursos ofrecidos por el Ayuntamiento de Barcelona. Ellas vinieron desde Bolivia (1), Brasil (3), Chile (1), Colombia (1), Ecuador (3), El Salvador (1), Paraguay (1), Perú (2) y Republica Dominicana (1). Ellas se encontraban en España de 8 meses hasta 20 años y tenían entre 23 y 45 años. Más de la mitad (12/14) tenía hijos(as).

Las mujeres inmigrantes concedieron entrevistas voluntariamente en Barcelona, España, con consentimiento informado. Teniendo en cuenta los planteamientos éticos establecidos por la American Psychological Association, se utiliza la sigla "P", queriendo decir con ello participante seguida del número de la participante con el objetivo de garantizar que la identidad de las participantes en el estudio fuese protegida.

Para analizar los datos obtenidos a través de las entrevistas semi estructuradas, se utilizó la técnica de la teoría fundamentada (Strauss \& Corbin, 2002), pues este abordaje permite comprender los significados que las participantes atribuyen a la experiencia de padecer y enfrentar el maltrato en un país ajeno al suyo.

\section{Resultados y discusión}

En esta sección se analizan las estrategias de las participantes para enfrentar la situación de violencia de género en la pareja y se divide los datos cualitativos obtenidos en dos categorías: factores de protección facilitadores del proceso de enfrentamiento de la violencia y factores de riesgo obstaculizadores del proceso de enfrentamiento de la violencia de género en la pareja.

\section{Factores de protección facilitadores del proceso de enfrentamiento de la violência}

Estrategias de enfrentamiento. Esta sub-categoría se refiere a las estrategias desplegadas por las participantes para hacer frente a la situación de violencia de género en 
la pareja en la experiencia migratoria como la búsqueda de ayuda social, búsqueda de ayuda institucional y reevaluación. Carver y Scheier (1989) definen la búsqueda de apoyo social como estrategia de enfrentamiento enfocada en el problema. A través de ella se considera que se puede reducir el impacto negativo de la violencia, como se ilustra a continuación: "Entonces cuando él se enteró que me tío(a) vivía ahí, pues le tenía más respeto porque sabe que mi tío(a) no es una persona joven (...) es una persona mucho mayor" (P.3).

El ejemplo evidencia que al pedir ayuda a su familiar, este(a) se fue a vivir con ella y, con ello, la ex pareja maltratadora dejó de ir a su casa a atormentarla. Dicho ejemplo pone en evidencia la importancia de las redes familiares para proveer apoyo emocional y facilitar recursos materiales, puesto que esas participantes contaban con el apoyo económico de la familia para compartir gastos de alquiler y comida.

Hay que tener en cuenta que ni todas mujeres cuentan con apoyo en España debido a la ruptura con las redes sociales en el país de origen. Si en el país de origen estaban acostumbradas a recurrir a la red para buscar el apoyo social y resolver sus problemas, cabe cuestionar, siguiendo a Carver y Scheier (1989), cómo lo hacen en un nuevo contexto donde esos recursos no están disponibles. La carencia del apoyo social puede sobrecargar las mujeres debido a la falta de personas que les encorajen (Kocot \& Goodman, 2003). Ello estuvo presente en el caso de cinco de las entrevistadas que no contaban con sus redes familiares en España. Ante esta situación, Agoff, Rajsbaum y Herrera (2005) señalan que es posible que las mujeres acudan a los servicios por no tener redes sociales a las que recurrir. Sin embargo, otro grupo de cinco participantes tenía algún familiar en España, pero ellos no fueron capaces de ayudarlas por no vivir cerca de Barcelona o por hacer que revivieran la vivencia de victimización, lo que sugiere, siguiendo a Ramírez, Santiago, y Hernández-Rosete (2005), que este grupo puede buscar ayuda institucional cuando las redes sociales no pueden atender a sus necesidades.

Se presentan ejemplos desde las voces de las mismas participantes sobre las estrategias de búsqueda de ayuda institucional:

Fue porque yo mismo puse de mi parte, porque a lo mejor, si yo no hubiera contado a mi médico de cabecera lo que me pasaba, yo no hubiera ido al (se refiere al nombre de la institución), yo fui, fui porque querría ir, porque querría salir de eso. (P.7)

Eu acho que é a determinação. Eu sempre pensei "Eu preciso sair. Eu sou (.) eu tô sozinha.”. (...) Eu tenho que arrumar um trabalho, primeiro eu tenho que fazer os meus papéis. Tenho que ter condições de vida pra essas crianças, uma escola boa (...) Peguei um papel e comecei a anotar o que eu tinha que fazer, as minhas metas. (P.14)

Romper el silencio al contar a su médico, buscar un trabajo y resolver problemas como arreglar la situación jurídica son estrategias enfocadas en el problema, pues las participantes invirtieron esfuerzos para alterar la fuente de estrés (maltrato y regularización de la situación jurídica) y para minimizar los efectos de dicha situación (ansiedad, depresión) a través de la búsqueda de ayuda institucional.
De igual manera, se puede decir que doce de las participantes intentaron enfrentar la violencia de género en la pareja buscando la solución del problema mediante el uso de los servicios sanitarios, sociales y legales. A través de ellos, pudieron acceder a asesoramiento jurídico, a tratamiento psicoterapéutico y a recursos externos (becas, guardería) que fueron factores facilitadores en el proceso de búsqueda del cese o reducción del maltrato. A esto se une el hecho de que regularizar la situación jurídica y buscar empleo, según contó la participante catorce, fueron los primeros pasos importantes para acceder a la independencia económica, lo que constituye un criterio importante para desvincularse del agresor.

Gadoni-Costa y Dell'Aglio (2011) afirman que asumir responsabilidad y solucionar problemas son estrategias de enfrentamiento enfocadas en el problema. Este dato de la literatura coincide con los hallazgos del estudio, pues las participantes dieron pasos activos para combatir el problema al poner de su parte como dijo la participante siete y dar el primer paso como comentó la participante uno. Dar el primer paso es salir de la posición de víctima como bien explican Cerruti y Rosa (2008) y poner de su parte es responsabilizarse por la recuperación, como propone Herman (2004). Ante este contexto, Albertin (2009) propone que las mujeres que han vivido violencia de género en la pareja salgan de la identidad de víctima y asuman la responsabilidad de entender el daño que se les ha causado y del que no tienen culpa.

Otro elemento que se debe considerar en el análisis de las estrategias de enfrentamiento es la presencia del apoyo social y/o institucional, pues según contaron diez de las entrevistadas, la presencia y consejo de amigas y/o familiares o la orientación de personas profesionales les animaron a tomar una decisión ante la situación de violencia de género en la pareja. A esto se une el hecho de que en el momento de interponer la denuncia, siete participantes estuvieron acompañadas de familiares o amigas.

Los datos apoyan el punto de vista de Waldrop y Resick (2004) al afirmar que el apoyo social es una estrategia de enfrentamiento, pues al recibir respuesta positiva por parte de la familia o de las personas profesionales, algunas participantes tomaron el primer paso para poner límites a la situación de violencia, ya que tenían sus necesidades de apoyo emocional y recursos materiales cumplidos (beca comedor ${ }^{1}$ ).

Además de las estrategias enfocadas en la resolución de problemas, ocho participantes adoptaron la estrategia de enfrentamiento enfocadas en la emoción como reevaluación. A modo ilustrativo, la participante seis reevalúo aspectos importantes de sí misma al recordar qué había buscado en la vida y que había tanta gente que la valoraba y valoró actitudes del compañero agresor cuando se dio cuenta de que "esa parafernalia de tu eres tonta no era más que la consciencia interna suya de que, de que era más débil". Se deduce que la estrategia de enfrentamiento de reevaluación puede también representar la etapa de recuperar la capacidad crítica descrita por Hirigoyen (2006) para salir del dominio en la cual la mujer percibe que la conducta violenta del agresor ofusca las debilidades del mismo. 
Otro ejemplo de estrategia de reevaluación es el establecimiento de límites de grado de violencia tolerable, como hizo la participante diez "Eu só sei que eu tomei essa decisão de terminar com ele, de denunciar e acabar, foi por causa da agressão que ele me fez". En este ejemplo, la participante percibió lo que Matos (2006) define como la naturaleza criminal de la violencia. Esta autora explica que otro ejemplo de reevaluación es reconocer que nadie tiene el derecho de maltratarla.

Otro ejemplo de reevaluación propuesto por Zink et al. (2006) es establecer autonomía respecto a la pareja, viviendo separado del compañero autor de la violencia o decretando rutinas en la casa que disminuyan el contacto entre la pareja. A modo de ejemplo, la participante siete reevaluó aspectos importantes de sí misma al tomar consciencia de que "mi salud se estaba deteriorando, que yo no aguantaba más" y eso la motivó a pedir el divorcio y estableció una rutina que redujo el contacto con la ex pareja al pasar a dormir en habitación diferente de él y mantener el móvil siempre en las manos: "Yo llegaba de mi trabajo, me preparaba rápido la cena, y el móvil y las llaves siempre en el bolsillo (...)Y con el móvil siempre en las manos para llamar a la policía pues si me hacia algo" (P.7).

Otras estrategias de enfrentamiento de reevaluación identificadas en los relatos que promovieron la reducción de contacto son salir del hogar e ir a una pensión, cambiarse de domicilio, como por ejemplo lo hizo la participante nueve: "me cambié de dirección, me fui a casa, a casa de esta amiga, me cambié de teléfono, me cambié, no sabe dónde yo vivo, lo eliminé del messenger, del facebook".

Con este recorrido, se pudieron identificar las diferentes estrategias desplegadas por el conjunto de 14 mujeres inmigrantes latinoamericanas enfrentar la violencia de género en la pareja en Barcelona, España. De todo ello, se deben extraer argumentos para repensar que no hubo estrategias buenas o malas, sino que sus acciones dependieron del contexto en que tuvo lugar la violencia y que ellas cambiaron al paso que las amenazas se alteraron. Sin embargo, el uso de la estrategia de enfrentamiento enfocada en la resolución del problema dependió de la accesibilidad a los servicios sociales y a la disponibilidad del apoyo social.

En este punto, cabe mencionar a Yoshihama (2002), que explica que la cultura influye en la elección de estrategias de enfrentamiento, pues en algunas culturas el uso de estrategias activas como denunciar la violencia puede ser desestimulada debido a las creencias culturales. En este sentido, esta estrategia no es adecuada en todas las situaciones, pues puede implicar peligrosidad si la mujer no fue asesorada. Por ello, la autora subraya que se debe arrojar luz no sólo al tipo de estrategia utilizada, sino también al significado del empleo de la estrategia en cada contexto. Los testimonios corroboran el mito de que las mujeres que padecen maltrato son pasivas, pues se puede observar toda la odisea que viven al enfrentarse a los obstáculos de la inmigración y violencia.

Hasta aquí se ha trabajado sobre los factores facilitadores que favorecen a la salida de la relación de la violencia hacia la recuperación. Sin embargo, Alencar-Rodrigues y Cantera (2013) comparan el proceso de dejar una relación de maltrato a un laberinto donde hay encrucijadas como los factores de riesgo obstaculizadores del proceso de enfrentamiento de la violencia de género en la pareja que se comentan a continuación.

\section{Factores de riesgo obstaculizadores del proceso de enfrentamiento de la violencia de género en la pareja}

En esta categoría se evidencia un conjunto de convicciones acerca del origen del maltrato, así como los sentimientos expresados por las entrevistadas que actuaron como obstaculizadores en el proceso de enfrentamiento de la violencia de género en la pareja.

Factores emocionales. Esta sub-categoría señala que el estado de ánimo de las participantes fue un factor que dificultó la adecuada respuesta al problema de la violencia de género en la pareja. Entre los elementos de análisis que constituyeron esta sub-categoría, se identificaron: la soledad, el miedo y la vergüenza.

La soledad es un tema presente en los testimonios de las entrevistadas por constituir una de las características del contexto migratorio (la ruptura de las redes sociales en el país de origen y la escasez de redes de apoyo en el país de recepción). Pero ahora parece conveniente acotar con mayor precisión la relación entre la soledad y la violencia de género en la pareja: "Si hubiese tenido más apoyo, porque el(la) niño(a) esté cuidada por una familiar, entonces me ha refugiado mucho en él (P.2)"; "Las veces que he vuelto a recaer es cuando me he sentido sola, he vuelto con él (...)porque no tenía, o sea lo veía como, como una familia, un refugio. (P.9)"

Aquí interesa resaltar que la soledad derivada de la escasez de redes sociales configuró un factor potencialmente de riesgo de la violencia de género en la pareja, pues se encontraron en una situación de aislamiento dónde la pareja pudo ser la única persona con quien pudieron contar en el país de recepción. Esos datos apoyan el punto de vista de Agoff, Rajsbaum y Herrera (2005), quienes afirman que el aislamiento es una condición de vulnerabilidad relacionada con los malos tratos en la pareja que puede propiciar la aparición o favorecer la perpetuación.

La soledad está presente en la historia de siete participantes y este dato invita a preguntarse ¿Quién sostiene a esas mujeres en esos procesos de adaptación y vivencia de la violencia? Ello apunta la necesidad de intervenciones comunitarias que promuevan la creación de redes de apoyo entre el colectivo inmigrante en la línea de lo desarrollado por el Proyecto Entre Vecinas del Ayuntamiento de L' Hospitalet. Esta propuesta, conforme la Diputació de Barcelona (2010), busca fomentar el contacto y los vínculos entre mujeres de diferentes orígenes que viven en L' Hospitalet a través de reuniones y espacios de convivencia que facilitan información sobre los recursos de la ciudad y los derechos de la población femenina inmigrante.

Además, el miedo de las consecuencias de no seguir en la relación cobra visibilidad en los testimonios de nueve 
participantes. Por un lado, el miedo estuvo relacionado con la preocupación de hacerse cargo de los(as) niños(as) sin contar con la ayuda del compañero maltratador: "Tenía miedo, porque decía uy qué me van a hacer, me van a deportar, me van a enviar para allá, me van a meter presa, me van a quitar mi hijito(a) (P.3)”.

De esto fragmento, resulta importante señalar que romper la relación implicaba muchos riesgos como la ausencia o escasez de recursos económicos. La inseguridad económica puede ser un factor que impide la búsqueda de ayuda como apunta Bui (2003). Esta autora constató que la dependencia económica del agresor influenció la decisión de las mujeres inmigrantes vietnamitas de buscar ayuda en el sistema judicial.

El miedo de la participante tres de que "me van a quitar mi hijito(a)" parece hacer eco a los planteamientos de Mullender (2000) sobre la preocupación que las mujeres tienen de que los servicios sociales les separen de sus hijos(as). Hay un considerable debate acerca del cuidado de los(as) niños(as), pues por un lado, esta autora plantea que no se puede interpretar erróneamente la habilidad cuidadora de la madre sin tener en cuenta que dicha habilidad puede verse afectada por las consecuencias de la victimización y, por otro lado, López (2011) recuerda que la ley prioriza proteger los(as) indefensos(as) como los(as) menores.

Otra cuestión de interés fue el miedo de deportación que afectó la toma de decisión enfrentamiento pues redujo la posibilidad de acceder a los servicios especializados en proteger a las mujeres de violencia de género en la pareja, ya que Casas (2009) advierte que buscar ayuda supone visibilizar la situación jurídica. En este contexto, se debe tener presente que Casas (2009) explica que el desconocimiento de las leyes españolas puede ser usado por la pareja autora de violencia de género en la pareja para amenazarla sobre las consecuencias de la denuncia o la ruptura tal como lo afirmó la participante tres al decir: "Entonces él me decía tu sabes que si pones la denuncia, tu no vas a poder ter la nacionalidad y todo. Yo tenía miedo de que me echaran de aquí de España y todo".

El miedo también estuvo asociado al aumento de la violencia y de las amenazas.

Si vuelves conmigo no te haré nada", me decía, "Pero si no, yo te prometo que de este mes tú no te salvas, te vas a amanecer, amanecer por ahí tirada, muerta", me decía, "y nadie ni va a saber quién fue", no, yo le digo yo:"De que, van a saber, van a saber, yo voy a dejar una nota de quien, quien, quien me hizo esto". Pero el hecho de matarme me, me daba mucho miedo (...) como a cualquiera, creo. (P.11).

El análisis de datos pone en evidencia que la separación no siempre significa seguridad debido a la experiencia de amenaza y miedo que las participantes ocho y once siguieron sufriendo después de la ruptura de la relación de pareja, tal como lo muestran también los hallazgos de Matos (2006). En este sentido, el hecho de dejar la relación es sinónimo de "invitación" al aumento de violencia o muerte como apuntan los hallazgos del estudio de Hage (2006). Este resultado lleva a la convicción de la importancia de que esas mujeres sean asesoradas en el momento de separación, teniendo en cuenta el posible maltrato posterior a la ruptura por parte del agresor.
Este resultado también sugiere que el miedo es un factor que favorece la ambivalencia pues les lleva a adoptar conductas que parecen contradictorias (Lloret, 2007) como retirar la denuncia, como contó la participante dos: "Yo porque hacerlo un bien, porque según yo iba hacer bien retirando la denuncia". Este tipo de actitudes fue frecuente entre las mujeres inmigrantes vietnamitas entrevistadas por Bui (2003) dado que ellas manifestaron interés en que sus compañeros fuesen punidos, pero expresaron miedo en relación con las consecuencias negativas de la denuncia como prisión o la pérdida de trabajo.

Con la idea de abundar en la relación entre cultura patriarcal y violencia de género en la pareja, se pasa a explorar el tema de las creencias dado que la experiencia muestra que no es posible realizar cambio alguno sin comprender el sistema de creencias de las mujeres que viven o han vivido violencia de género en la pareja, puesto que estas pueden estar obstaculizando la permanencia en los servicios y la salida de la violencia.

Creencias. Las creencias se refieren a los significados sobre la naturaleza y el funcionamiento de los papeles y conductas del sexo masculino y femenino y sus relaciones de pareja, de familia, creencias sobre la violencia, el amor y que reflejen la relación en el comportamiento humano (Roca-Cortés, Espín, Rosich, Cantera, \& Strey, 2005; Soriano, 2007). Las expectativas de género sobre las mujeres y hombres establecidas por la cultura patriarcal contribuyen a la subordinación de las mujeres (Roca-Cortés et al., 2005; Soriano, 2007).

Cabe destacar que las creencias culturales y religiosas representadas por "Yo decía que era mi cruz" configuran, según diferentes teóricos(as) (Ayyub, 2000; Bhuyan, Mell, Senturia, Sullivan, \& Shiu-Thornton, 2005), barreras a la hora de romper con la violencia de género en la pareja. Se puede inferir que lo expuesto por la participante "yo decía qué habrá hecho en la vida para merecerme esto" refleja los planteamientos de autores(as) como Bhuyan et al. (2005) y Shirwadkar (2004) sobre creencias que atribuyen la violencia de género en la pareja a una falta de la mujer. Tal como sucede en los hallazgos de Bhuyan et al. (2005) sobre mujeres indias en Canadá que aceptan que su marido ejerza control sobre ellas para evitar generar conflictos, la participante uno, que "hacía todo que él querría para que él estuviera contento”, también lo intentaba sometiéndose a su compañero.

Las creencias culturales relacionadas con la preservación de la familia y la educación de los(as) hijos con los padres, presentadas en las narrativas de cinco entrevistadas, indican que los valores culturales normativos del país de origen son idealizados y romantizados, como señalan Runner, Yoshihama y Novick (2009). Sobre la base de estos supuestos, se puede inferir que es menos probable que las mujeres inmigrantes busquen ayuda en los servicios sociales debido a la creencia en la preservación de la "familia estable", tal y como plantean Bhuyan et al. (2005).

Tener en cuenta estos planteamientos implica partir de una perspectiva de género que arroja luz sobre la socialización de las mujeres construidas sobre los pilares del cuidado del otro, 
del perdón y del sacrificio. Siguiendo este planteamiento, cabe preguntarse si algunas participantes a coste de mucho sufrimiento sintieron pena del compañero, como la participante diez, y le perdonaron, como lo hicieron cuatro entrevistadas, por seguir teniendo esperanza en que la relación cambiara a fin de lograr la idealizada "familia estable" inculcada mediante las creencias patriarcales.

También conviene detectar creencias culturales en relación con el amor romántico, pues según Lloret (2007, p. 48), tienen "una fuerte influencia en la configuración del psiquismo femenino y en la regulación de las relaciones de pareja". Así, la aceptación de esas creencias dificulta el discernimiento del vínculo violento, como bien se muestra a través de los relatos de las siguientes participantes: "Estaba súper enamorada, súper ciega" (P.1) o "Cuando uno se enamora no se da cuenta de nada y a mí me pasó eso con él. Me celaba, pero mira bueno, yo mirara en plan de broma" (P.3).

Otro ejemplo de creencias patriarcales fue "Que si el marido nos dice algo, no hablamos" refleja la construcción de la feminidad basada en la sumisión e inferioridad, la cual llevó a la participante tres a aceptar que su pareja ejerciera el control sobre ella para evitar conflicto en la relación. Así, la educación patriarcal refuerza el hecho de aceptar la dominación masculina con lo cual se reprime la reivindicación de derechos y oposición contra la opresión social. Además su comentario "tenemos que soportarlo, aguantarlo o lo que él diga hacerlo" dio a entender que la sociedad de origen les educó para tener paciencia y aceptar la situación, lo que apoya lo encontrado en el estudio de Ayyub (2000) con mujeres inmigrantes surasiáticas en Estados Unidos. Este factor pudo impedir la búsqueda de ayuda en el país de recepción, dado que en su país la violencia contra la mujer se percibe como algo normal.

Parece conveniente detenerse en el fragmento "claro que sufría", pues eso hace eco a los planteamientos de Mullender (2000), al reiterar que aunque las mujeres hayan sido educadas en un sistema de valores sobre el cual la familia es sagrada no quiere decir que a ellas les resulte menos doloroso aguantar la violencia. Todo eso lleva a apoyar también a Jacobson y Gottman (2001), quienes plantean que algunas mujeres pueden haber sido socializadas en entornos donde los hombres maltrataban a las mujeres, por lo que tal vez no sepan que merecen algo mejor o que haya algo diferente de la violencia.

Cabe destacar que el hecho de justificar la violencia mediante procesos psicológicos de racionalización como "es que su padre era como él" (P.6) y "yo pienso que es eso la raíz de lo que te has criado tú aprendes y yo pienso que él quiere hacer lo mismo" (P.4), el hecho de buscar entender la violencia de género en la pareja a través de explicaciones como el uso de alcohol o el haber sido testigo y/o víctima de violencia en la infancia fueron factores que obstaculizaron el proceso de búsqueda de ayuda.

Estos datos empíricos confirman lo encontrado en el estudio de Agoff et al. (2006), quienes subrayan que el análisis de la percepción subjetiva de las mujeres sobre los motivos de violencia de género en la pareja permite entender las acciones que ellas adoptan para dar una respuesta al problema de la violencia. Según Agoff et al. (2006) y Lloret (2007), buscar causas como el uso de alcohol y los problemas en la infancia de la pareja para explicar la violencia de género en la pareja puede desresponsabilizar en parte a la pareja de su conducta violenta. Estas constataciones llevan a un planteamiento que implica que son necesarias intervenciones que tengan en cuenta la desconstrucción de creencias patriarcales y sobre las causas de la violencia, puesto que esas creencias actúan como dificultadoras o facilitadoras de la recuperación de la relación de violencia. Así, la revisión de las creencias brinda la toma de consciencia de la educación patriarcal, de la opresión personal en un contexto de opresión social de todas las mujeres y, a la vez, facilita que las mujeres conecten con su fortaleza psicológica y se enfrenten a la relación de maltrato (Soriano, 2007). En este marco, Cerruti y Rosa (2008, p. 1057) explican que resulta necesario que las mujeres se disloquen de la posición de víctima en la cual ellas recurren a los hechos (causas de la violencia) y "atribuyen al otro la responsabilidad por aquello que le hace sufrir" y adopten otra posición en la cual se responsabilicen por su deseo.

\section{Conclusión}

El cese o reducción de la violencia de género en la pareja se caracterizó por un proceso que suele ser largo, que contempla muchos intentos de buscar ayuda en servicios institucionales distintos y que está afectado por los recursos de las mujeres y del contexto en el que se encuentran. Se puede identificar que las participantes han utilizado diferentes medios para hacer frente a la violencia.

Tanto en el presente estudio como en la literatura consultada, las construcciones culturales acerca de la preservación de la familia y del rol de la mujer son barreras a la hora de enfrentar el maltrato. Así el contexto migratorio puede comportar que las mujeres inmigrantes se prendan a sus creencias tradicionales y a sus formas de actuar, dificultando la posibilidad de desmarcarse de la cultura de origen y de no integrar aquello de la cultura del contexto de recepción que le parezca apropiado, desde una mirada crítica. Dicha situación señala la necesidad de un espacio donde las mujeres puedan discutir sus valores culturales e identificar aquellos que les ponen en situación de riesgo a la violencia de género en la pareja. Además el miedo a la deportación obstaculiza el cese o la reducción a la violencia de género en la pareja es una cuestión en la cual las políticas públicas deben incidir no sólo garantizando en la ley el derecho de no deportación, sino también asegurando que las mujeres inmigrantes reciban esta información antes de decidir si denuncian a su agresor.

El aislamiento puede ser causa o consecuencia de la violencia de género en la pareja, con lo cual cualquier mujer que viva violencia de género en la pareja puede no contar con una red social. No obstante, en el caso de las mujeres inmigrantes, las redes sociales pueden ser aún más limitadas debido a la ruptura de las redes en el país de origen, a la situación de 
violencia. Con este estudio se pretende no sólo remarcar que el aislamiento es un factor obstaculizador para la salida de la violencia, sino también poner de relieve que la soledad generada por dicha situación es el punto en el cual se debe intervenir.

\section{Referências}

Agoff, C., Rajsbaum, A., \& Herrera, C. (2006). Perspectivas de las mujeres maltratadas sobre la violencia de pareja en México. Salud Pública de México, 48(2), 307-314. Recuperado de http://www.scielo.org.mx/scielo. php?script=sci_arttext\&pid=S0036-36342006000800011\&lng=es\&tlng=es.

Alberdi, I. (2005). Cómo reconocer y cómo erradicar la violencia contra las mujeres. En Violencia: Tolerancia Cero. Programa de prevención de la Obra Social "la Caixa". Barcelona: Fundación La Caixa.

Albertín, P. (2009). Mujeres inmigradas que padecen violencia en la pareja y sistema socio jurídico: Encuentros y desencuentros. Portularia, 9(2), 1734.

Alencar-Rodrigues, R., \& Cantera, L. (2013). Del Laberinto hasta la recuperación de la violencia. Interamerican Journal of Psychology, 47(1), 61-70. Recuperado de http://journals.fcla.edu/ijp/article/view/77392

Antoniazzi, A. S., Dell'Aglio, D., \& Bandeira, D. R. (1998). O conceito de coping: Uma revisão teórica. Estudos de Psicologia, 3(2), 273-294. doi: 10.1590/ S1413-294X1998000200006

Ayyub, R. (2000). Domestic violence in the south Asian muslim immigrant population in the United States. Journal of Social Distress and the Homeless, 9(3), 237-248. doi: 10.1023/A:1009412119016

Bhuyan, R., Mell, M., Senturia, K., Sullivan, M., \& Shiu-Thornton, S. (2005). "Women must endure according to their karma": Cambodian immigrant women talk about domestic violence. Journal of Interpersonal Violence, 20(8), 902-921. doi: 10.1177/0886260505277675

Bui, H. N. (2003). Help-seeking behavior among abused immigrant women: A case of vietnamese american women. Violence Against Women, 9(2), 207239. doi: $10.1177 / 1077801202239006$

Cantera, L. M. (2005). Violencia en la pareja: Fenómenos, procesos y teorías. In T. Sánchez (Org.), Maltrato de género, infantil y de ancianos (pp.55-94). Salamanca: Publicaciones Universidad Pontificia de Salamanca.

Cantera, L. M. (2007). Casais e violência: Um enfoque além do gênero. Porto Alegre: Dom Quixote.

Carver, C. S., \& Scheier, M. F. (1989). Assessing coping strategies: A theoretically based approach. Journal of Personality and Social Psychology, 56(2), 267-283. doi: 10.1037/0022-3514.56.2.267

Casas, M. J. B. (2009). Repercusiones psicológicas en las víctimas de la violencia de género: las víctimas inmigrantes. Crítica, 59, 960, 39-43.

Cerruti, M. Q., \& Rosa, M. D. (2008). Em busca de novas abordagens para a violência de gênero: A desconstrução da vítima. Revista Mal-Estar e Subjetividade, 8(4), 1047-1076. Recuperado de http://pepsic.bvsalud.org/scielo.php?script=sci_ arttext\&pid=S1518-61482008000400009\&lng=pt\&tlng=pt.

Conferencias de las Naciones Unidas sobre la Mujer (1995). Nuevas medidas e iniciativas para la aplicación de la declaración y la plataforma de acción de Beijing. Recuperado de www.cinu.org.mx/temas/mujer/confmujer.htm

Cortés, N. R., \& Serra, J. M. (2011). Intervención grupal en violencia sexista: Experiencia, investigación y evaluación. Barcelona: Editorial Herder.

Diputació Barcelona (2010). Un proyecto de L'Hospitalet de Llobregat crea punetes entre mujeres autóctonas e inmigradas. Recuperado de http://www. diba.cat/butlletins/detallRevista.asp?id=14916

Folkman, S., Lazarus, R. S., Gruen, R. J., \& DeLongis, A. (1986). Appraisal, coping, health status and psychological symptoms. Journal of Personality and Social Psychology, 50, 571-579.

Gadoni-Costa, L. M., \& Dell’Aglio, D. D. (2009). Mulheres em situação de violência doméstica: vitimização e coping. Revista Interinstitucional de Psicologia, 2(2), 151-159.
Gadoni-Costa, L. M., \& Dell'Aglio, D. D. (2011). Multigeracionalidade e o proceso de enfrentamento da violência contra a mulher: Estudo de caso. Salud \& Sociedad, 2(1), 63-78. Recuperado de http://dialnet.unirioja.es/ servlet/articulo? codigo $=4017557$

Hage, S. M. (2006). Profiles of women survivors: The development of agency in abusive relationship. Journal of Counseling \& Development, 84(1), 83-94.

Heilborn, M. L. (1998). Gênero: Um olhar estruturalista. In J. Pedro \& M. Grossi (Orgs.), Masculino, feminismo, plural: gênero na interdisciplinariedade (pp. 43-55). Florianópolis: Editora Mulheres.

Herman, J. (2004). Trauma y recuperación: Cómo superar las consecuencias de la violencia. Madrid: Editorial Espasa Calpe.

Hirigoyen, M. (2006). Mujeres maltratadas: Los mecanismos de la violencia en la pareja. Barcelona: Paidós.

Jacobson, N., \& Gottman, J. (2001). Hombres que agreden a sus mujeres: Cómo poner fin a las relaciones abusivas. Barcelona: Paidós.

Kocot, T., \& Goodman, L. (2003). The roles of coping and social support in battered women's mental health. Violence Against Women, 9(3), 323-346. doi: 10.1177/1077801202250075

Lazarus, R. S. (1993). Coping theory and research: Past, present, and future. Psychosomatic Medicine, 55, 234-247.

Lloret, I. (2007). La violencia contra las mujeres en la pareja. In E. Gil \& I. Lloret (Orgs.), La violencia de género (pp. 39-53). Barcelona: Editorial UOC.

López, E. (2011, Abril). Violencia de género en procesos de separación. Conferencia plenaria presentada en el VI Congreso de Psicología Jurídica y Forense de la Sociedad Española de Psicología Jurídica y Forense, Palma de Mallorca, España.

Louro, G. (1997). Gênero, sexualidade e educação: Uma perspectiva pósestruturalista. Petrópolis: Vozes.

Matos, M. A. V. (2006). Violência nas relações de intimidade: Estudo sobre a mudança psicoterapéutica na mulher (Tese de doutorado não-publicada. Universidade do Minho, Porto).

Mullender, A. (2000). La violencia doméstica: Una nueva visión de un viejo problema. Barcelona: Paidós.

Ramírez, C., Santiago, R., \& Hernández-Rosete, D. (2005). La percepción del apoyo social en mujeres con experiencia de violencia conyugal. Salud Mental, 28(4), 66-73. Recuperado de http://www.redalyc.org/ pdf/582/58242807.pdf

Roca-Cortés, N., Espin, J., Rosich, M., Cantera, L. M., \& Strey, M. N. (2005). Cambios de creencias e intervención en mujeres que sufren maltrato. Revista Electónica de la Universitat Jaume I, 1(1), 143-155.

Runner, M., Yoshihama, M., \& Novick, S. (2009). Intimate partner violence in immigrant and refugee communities: Challenges, promising practices and recommendations. Recuperado de http://www.rwjf.org/files/research/ ipvreport20090331.pdf

Señas (2006). Diccionario para la enseñanza de la lengua española para brasileños. São Paulo: Martins Fontes.

Shirwadkar, S. (2004). Canadian domestic violence policy and Indian immigrant women. Violence Against Women, 10(8), 860-879. doi: $10.1177 / 1077801204266310$

Soriano, M. (2007). ¿Es necesario un enfoque teórico-técnico o es precisa también una perspectiva de género? Necesidades actuales de investigación en violencia. Recuperado de http://www.majeso.es/publicacions.html

Strauss, A., \& Corbin, J. (2002). Bases de la investigación cualitativa. Técnicas y procedimientos para desarrollar la teoría fundamentada. Medellin: Editorial Universidad de Antioquia.

Waldrop, A. E., \& Resick, P. A. (2004). Coping among adult female victims of domestic violence. Journal of Family Violence, 19(5), 291-302. doi: 10.1023/B:JOFV.0000042079.91846.68

Yoshihama, M. (2002). Battered women's coping strategies and psychological distress: Differences by immigration status. American Journal of Community Psychology, 30(3), 429-452. 
Zink, T., Jacobson, C. J., Pabst, S. Regan, \& Fisher, B. S. (2006). A lifetime of intimate partner violence: Coping strategies of older women. Journal of Interpersonal Violence, 21(5), 634-651. doi: 10.1177/0886260506286878

\section{Nota}

1. Beca comedor pretende atender situaciones de necesidad económica o desamparo. Están dirigidas exclusivamente a alumnos matriculados en estudios oficiales que sean nacionales de un estado miembro de la Unión Europea o, en caso de extracomunitarios, tengan la condición de residentes en España. Dan derecho a vales de comida.

Roberta de Alencar-Rodrigues, Doutora em Psicologia Social pela Universidade Autônoma de Barcelona (UAB), é Professora, Supervisora de Estágio e Orientadora de Trabalho de Conclusão de Curso da Escola de Psicologia da Faculdade Meridional (IMED). Endereço para correspondência: Rua Senador Pinheiro, 304. Cep: 99070-220,Passo Fundo - RS. Telefone: (54) 30459018. E-mail: roberta.rodrigues@imed.edu.br

Leonor María Cantera Espinosa, Doutora em Filosofia pela Universidade de Porto Rico (UPR), Doutora em Psicologia Social pela Universidade Autônoma de Barcelona (UAB), é Professora Titular do Departamento de Psicologia Social da Faculdade de Psicologia da Universidade Autônoma de Barcelona (UAB) e Diretora do Departamento de Psicologia Social da Universidade Autônoma de Barcelona (UAB). E-mail: leonor. cantera@uab.es 